\title{
Tinea capitis mimicking alopecia areata
}

\section{Saber Dooqaei Moqadam¹, Ramina Mofarrah², Kousar Jahani Amiri', Fatemeh Montazer ${ }^{3}$, Anahita Barqi ${ }^{1}$, Ramin Mofarrah ${ }^{4}$}

\author{
${ }^{1}$ Student Research Committee, Sari Branch, Islamic Azad University of Medical Sciences, Sari, Iran, ${ }^{2}$ Student Research \\ Committee, Shiraz Branch, University of Medical Sciences, Shiraz, Iran, ${ }^{3}$ Department of Pathology, Iran University of \\ Medical Sciences (IUMS), Tehran, Iran, ${ }^{4}$ Department of Dermatology, Faculty of Medicine, Sari Branch, Islamic Azad \\ University of Medical Sciences, Sari, Iran
}

Corresponding author: Dr. Ramin Mofarrah, E-mail: Ramin.Mofarrah@gmail.com

\begin{abstract}
Tinea capitis (TC) is the most common dermatophyte scalp infection in children and an unusual dermatophytosis in adults. The clinical appearance of tinea capitis is highly variable and depends on the causative organism, type of hair invasion, and degree of the host inflammatory response. The commonly observed features are patchy hair loss with varying degrees of scaling and erythema. The clinical signs may be subtle and diagnosis may be challenging. We report the case of an adult patient with tinea capitis mimicking alopecia areata. The patient was initially diagnosed with alopecia areata and completed one month of treatment without clinical benefits. In view of no clinical signs of tinea capitis, a biopsy was performed. A scalp punch biopsy revealed an endothrix dermatophytosis. The patient's medication was switched to $250 \mathrm{mg}$ terbinafine daily for 8 weeks and $2 \%$ ketoconazole shampoo. The patient completed two months of therapy with maintenance of hair regrowth and resolution of symptoms and scales.
\end{abstract}

Key words: Tinea capitis; Alopecia areata; Dermascopy

\section{INTRODUCTION}

Tinea capitis is a common scalp condition seen primarily in the pediatric population. It has been observed in postpubertal individuals, but is far less common, and can present bizarrely, often leading to misdiagnosis [1].

Its clinical manifestations range from mild scaling with little hair loss to large, inflammatory, and pustular plaques with extensive alopecia [2].

Delayed diagnosis and treatment can result in scarring alopecia [3].

We describe a case of endothrix tinea capitis mimicking alopecia areata.

\section{CASE REPORT}

A healthy and active 39-year-old woman presented to our dermatology clinic with a l-year history of a painless patch of alopecia associated with pruritus on the occiput of the scalp with no erythematous background.

There was no significant drug history. She was otherwise healthy, denied constitutional symptoms, and was not immunosuppressed.

A physical examination revealed a severe alopecia involvement of the occiput and vertex (Fig. 1). There was no warmth or erythema and scant scale.

Dermatoscopy showed multiple broken hairs, comma hairs, and cadaver hairs (Fig. 2).

The patient was initially diagnosed with alopecia areata and completed one month of treatment with clobetasol cream, zinc capsule, and intramuscular triamcinolone without clinical benefits. Repeated doses with intralesional injection of triamcinolone gave no response. In view of no clinical signs of tinea capitis, a biopsy was performed.

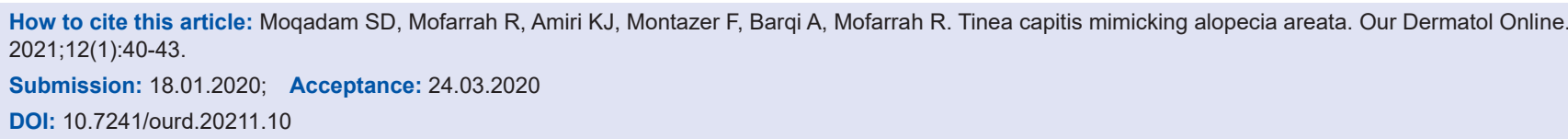


A $4 \mathrm{~mm}$ scalp punch biopsy revealed an acanthotic epidermis with a dilated follicle infundibulum, containing numerous fungal elements and occasionally invading into the hair shaft, diagnosed as an endothrix dermatophytosis (Figs. 3a and 3b). Perifollicular lymphocytic and neutrophilic infiltration was also observed.

The patient's medication was switched to $250 \mathrm{mg}$ terbinafine daily for 8 weeks and 2\% ketoconazole shampoo. She completed two months of therapy with maintenance of hair regrowth and resolution of symptoms and scales (Figs. 4 and 5).

\section{DISCUSSION}

Tinea capitis (TC) is a common scalp dermatosis in the prepubertal population, affecting ages 3-7 years. $\mathrm{TC}$ is rarely seen in postpubertal individuals, but has been described in this population and is reported to be more common in women [3].

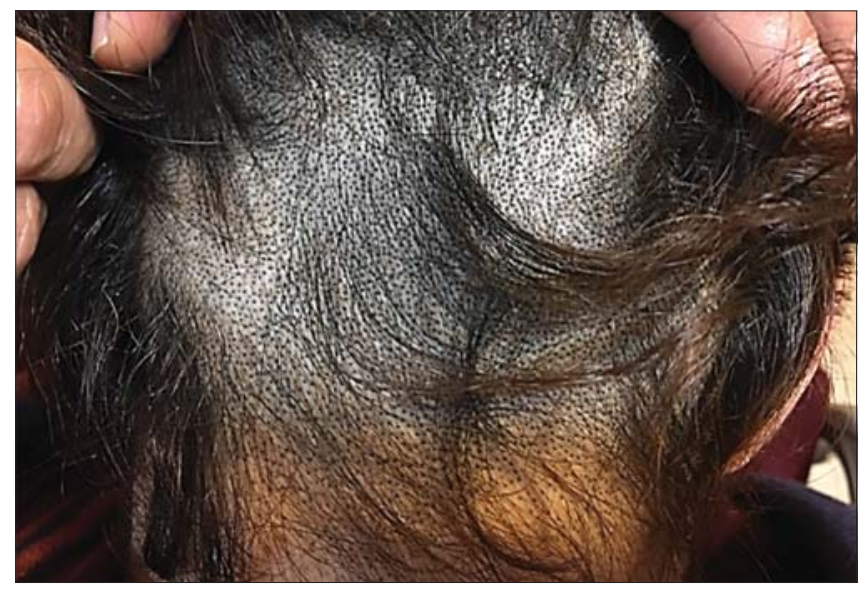

Figure 1: An alopecia involvement of the occiput and vertex.

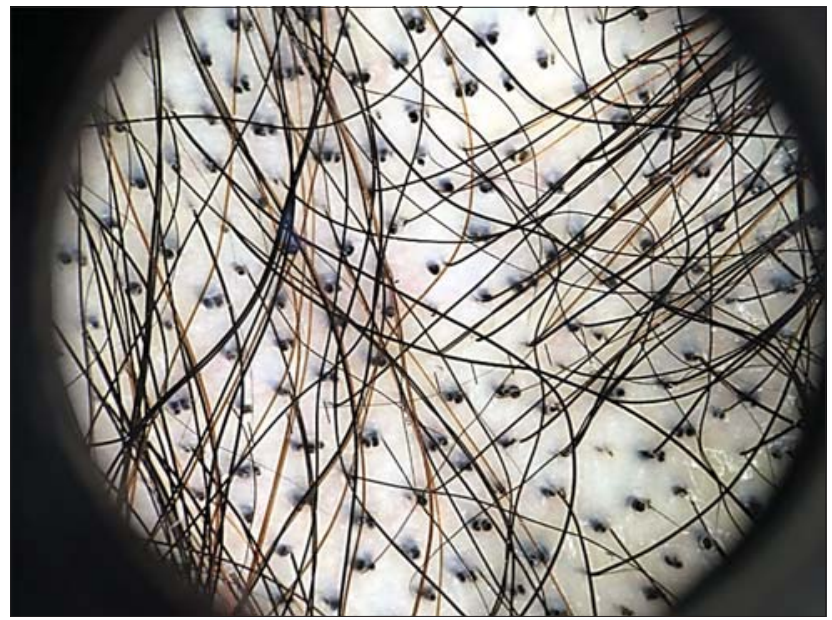

Figure 2: A dermoscopic image showing a comma sign and cadaver hairs.
TC is an unusual dermatophytosis in adults $(3-11 \%$ of all cases) and is, therefore, often misdiagnosed. It mostly affects postmenopausal women and immunosuppressed individuals, and, in the majority of cases, is due to anthropophilic dermatophytes, especially Trichophyton tonsurans and Trichophyton violaceum [4].

The clinical appearance of tinea capitis is highly variable and depends on the causative organism, type of hair invasion, and degree of the host inflammatory response. The common features are patchy hair loss with varying degrees of scaling and erythema. The clinical signs may be subtle and diagnosis may be challenging [1].

The endothrix type of the infection may be caused by $T$. tonsurans, Trichophyton soudanense, African members of the Trichophyton rubrum complex, T. violaceum, and rarely T. rubrum (Table 1 ).

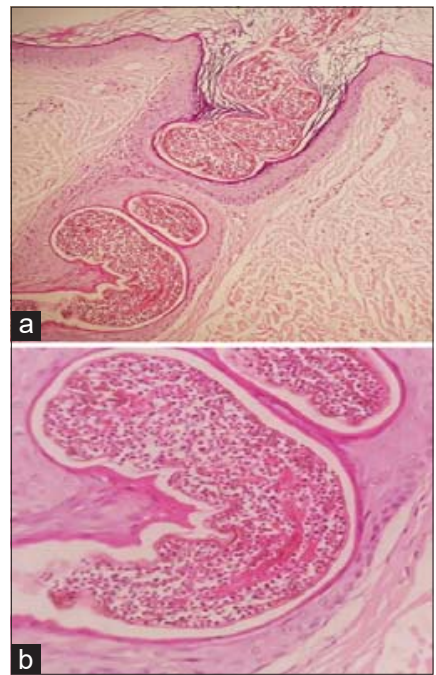

Figure 3: (a-b) Photomicrographs showing endothrix tinea capitis.

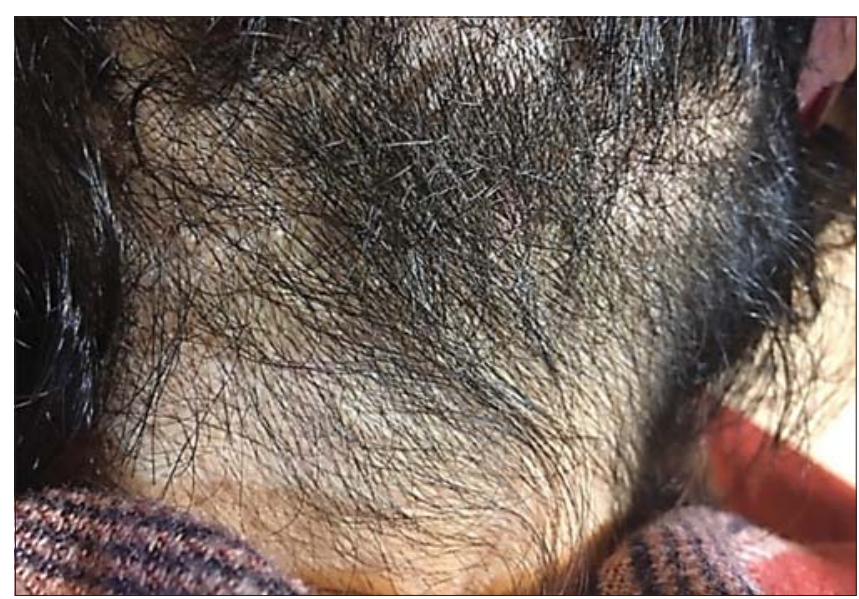

Figure 4: Hair regrowth one month after treatment. 


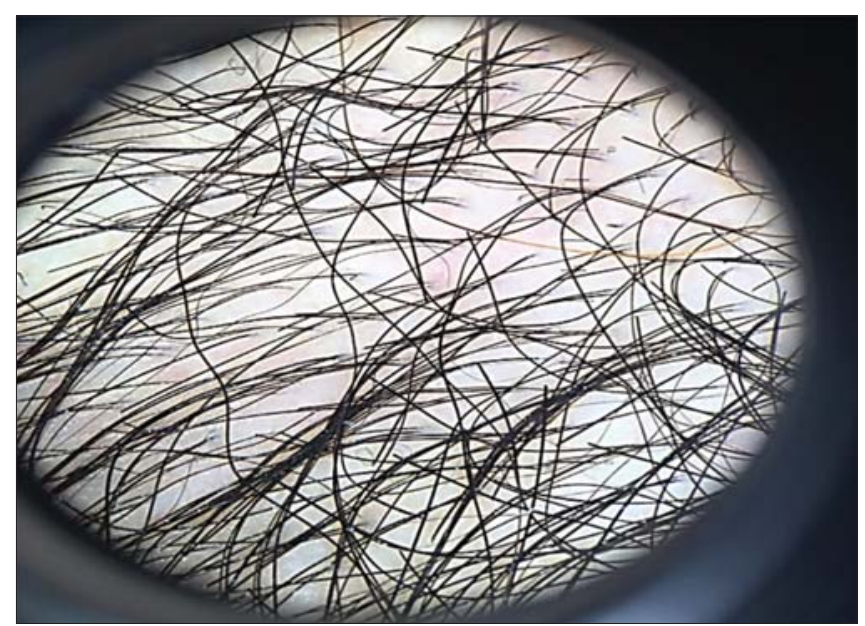

Figure 5: A dermoscopic image after treatment.

Table 1: Choice of drug depending on the organism isolated [1].

\begin{tabular}{ll}
\hline Organism & Choice of drug \\
\hline Trichophyton tonsurans & Terbinafine \\
Trichophyton violaceum, soudanense & Terbinafine \\
Microsporum canis & Griseofulvin or itraconazole \\
Microsporum audouinii & Griseofulvin or itraconazole \\
\hline
\end{tabular}

This type of infection is nonfluorescent under Wood's light. Hairs often break at the scalp level, leaving swollen hair stubs within the follicles (black dot ringworm) [2].

The condition should be projected in any patient with a solitary or numerous diminutive plaques of hair loss associated with cut hairs, scales, and itching [5].

Broken hairs that resemble black dots may or may not be present, and can be scattered among normal hairs or concealed by scale. The inflammatory type of tinea capitis exhibits marked edema, redness, pustules, nodules, or sinus tracts with purulent discharge and crusting. Hair loss may be patchy or involve the entire scalp. Inflammatory lesions may be tender with marked cervical lymphadenopathy and can be associated with systemic symptoms. Diagnosis is confirmed by Wood's lamp, microscopic detection of fungal elements by a $\mathrm{KOH}$ test of the hair shaft, histopathological evidencing of hyphae in hair follicles with a PAS stain, or species identification by a fungal culture [6] .

Alopecia areata incognita is a variety of alopecia areata characterized by acute diffuse shedding of telogen hairs without typical patches. Clinically, it has the features of telogen effluvium, but could also be misdiagnosed as alopecia androgenetica, which is the reason why a scalp biopsy is often required to confirm the clinical diagnosis [7].
The differential diagnosis of hair loss in children involves mostly TC. Slowinska et al. assert that comma hair is a distinguishing feature of TC, followed by broken and black dots (dystrophic hair). Comma hair, corkscrew hair, and pigtail hair were all observed only in patients with TC, thus embodying the characteristic features [8].

The differential diagnosis of tinea capitis is extensive and encompasses all conditions that may cause patchy hair loss, scaling, or scalp inflammation. Scalp psoriasis, seborrheic dermatitis, and atopic dermatitis may be difficult to differentiate from noninflammatory tinea capitis, although these conditions are usually more diffuse, and there may be characteristic signs elsewhere. Alopecia areata is generally non-scaly but may occasionally demonstrate erythema. Exclamation point hairs must be distinguished from the broken hairs of tinea capitis. Lupus erythematosus, lichen planopilaris, and trichotillomania, although relatively rare, should also be considered. Inflammatory tinea capitis variants may be misdiagnosed as bacterial folliculitis, folliculitis decalvans, or abscesses. Regional lymphadenopathy may be associated with the inflammatory variants of tinea capitis [1].

\section{CONCLUSION}

In conclusion, we describe a case of endothrix tinea capitis mimicking alopecia areata in dermoscopy, which showed cadaver hairs and a comma sign. Therefore, it should be kept in mind that tinea capitis can display the dermoscopic features of alopecia areata and can expand under incorrect treatment, as with corticosteroids.

\section{Consent}

The examination of the patient was conducted according to the principles of the Declaration of Helsinki.

The authors certify that they have obtained all appropriate patient consent forms, in which the patients have given consent for images and other clinical information to be included in the journal. The patients understand that their names and initials will not be published and due effort will be made to conceal their identity, but that anonymity cannot be guaranteed.

\section{REFERECES}

1. Fuller LC, Barton RC, Mohd Mustapa MF, Proudfoot LE, Punjabi SP, Higgins EM. British Association of Dermatologists' guidelines for the management of tinea capitis 2014. Br J Dermatol. 2014;171:454-63.

2. Hay RJ. Tinea capitis: current status. Mycopathologia. 2017;182:87-93. 


\section{www.odermatol.com}

3. Stein LL, Adams EG, Holcomb KZ. Inflammatory tinea capitis mimicking dissecting cellulitis in a postpubertal male: a case report and review of the literature. Mycoses. 2013;56:596-600.

4. Tangjaturonrusamee C, Piraccini BM, Vincenzi C, Starace M, Tosti A. Tinea capitis mimicking folliculitis decalvans. Mycoses. 2011;54:87-8.

5. Haliasos EC, Kerner M, Jaimes-Lopez N, Rudnicka L, Zalaudek I, Malvehy J, et al. Dermoscopy for the pediatric dermatologist part I: dermoscopy of pediatric infectious and inflammatory skin lesions and hair disorders. Ped Dermatol. 2013;30:163-71.

6. Goldsmith LA, Katz S, Gilchrest B, Paller A, Leffell D, Wolff K. Fitzpatrick's Dermatology in General Medicine. $8^{\text {th }}$ Edn: MC Graw Hill. 2012;2:2280.
7. Kinoshita-Ise M, Fukuyama M, Ohyama M. Color-transition sign: A useful trichoscopic finding for differentiating alopecia areata incognita from telogen effluvium. J Dermatol. 2018;45:e224-5.

8. Waśkiel-Burnat A, Rakowska A, Sikora M, Ciechanowicz P, Olszewska M, Rudnicka L. Trichoscopy of tinea capitis: a systematic review. Dermatol Ther (Heidelb). 2020;10:43-52.

Copyright by Saber Dooqaei Moqadam, et al. This is an open access article distributed under the terms of the Creative Commons Attribution License, which permits unrestricted use, distribution, and reproduction in any medium, provided the original author and source are credited.

Source of Support: Nil, Conflict of Interest: None declared. 